\title{
Influence du génotype sexuel sur les caractéristiques de la reproduction de femelles (génotype WZ) et de pseudofemelles (génotype ZZ) chez le tilapia Oreochromis aureus
}

\author{
Damien Desprez ${ }^{(*)}$, Charles Mélard
}

Laboratoire de démographie des poissons et d'aquaculture, Station d'aquaculture de Tihange, Université de Liège, Chemin de la Justice, 10, 4500 Tihange, Belgique.

Reçu le 6 février 1998 ; accepté le 25 mai 1998.

\begin{abstract}
Influence of sexual genotype on reproductive traits of females (genotype WZ) and pseudofemales (genotype ZZ) in the tilapia Oreochromis aureus. The sex reversal of Oreochromis aureus (male genotype: $\mathrm{ZZ}$; female genotype: WZ) with an oestrogen (17 $\alpha$-ethynylestradiol) treatment produces pseudofemales with a male genotype (ZZ). Crossing these fish with normal males (ZZ) gives, in theory, $100 \%$ male offspring. We have determined and compared the principal traits of reproductive biology of three broodstock batches of $O$. aureus. First batch (batch 1) was constituted using normal females only, the second (batch 2) involved pseudofemales only, while the third batch (batch 3) contained both females and pseudofemales. Fecundity and mean egg weight was not significantly different in females and pseudofemales. In the third group, spawning rate of the pseudofemales (0.6 spawns) was lower than that of the females (6.4 spawning). In this last group, $60 \%$ of the pseudofemales never reproduced. In batches 1 and 2, there were no differences between females ( 6.9 spawns) and pseudofemales ( 4.7 spawns). Lowest spawning rate of pseudofemales may result from the expression of the male sexual genotype of these fish. 0 Ifremer/Elsevier, Paris
\end{abstract}

\section{Hormonal sex-reversal / oestrogen / reproduction / fecundity / genotype / tilapia / Oreochromis aureus}

Résumé - L'inversion hormonale du sexe chez Oreochromis aureus (génotype mâle ZZ et génotype femelle ZW) avec un œstrogène ( $17 \alpha$-éthynylasstradiol) conduit à l'obtention de pseudofemelles, caractérisées par un genotype mâle (ZZ). Le croisement de ces pseudofemelles avec des mâles (ZZ) donne, en théorie, des populations $100 \%$ mâles. Nous avons déterminé et comparé les principaux paramètres caractéristiques de la reproduction au sein de trois groupes de géniteurs $O$. aureus. Le premier groupe (groupe 1) est composé de femelles, le second (groupe 2) de pseudofemelles et le troisième (groupe 3 ) de femelles et de pseudofemelles. La fécondité et le poids des oufs des femelles et des pseudofemelles ne sont pas significativement différents. Dans le groupe 3, le taux de reproduction des pseudofemelles ( 0,6 pontes) est plus faible que celui des femelles $(6,4$ pontes). Dans ce groupe $60 \%$ des pseudo-femelles ne se sont jamais reproduites. Dans les groupes 1 et 2 , il n'existe pas de différence entre les femelles (6,9 pontes) et les pseudofemelles ( 4,7 pontes). Le plus faible taux de reproduction des pseudofemelles pourrait résulter de l'expression du génotype sexuel mâle chez ces individus. (O) Ifremer/Elsevier, Paris

Inversion hormonale du sexe / oestrogène / reproduction / fécondité / génotype / tilapia / Oreochromis aureus

\section{INTRODUCTION}

Les tilapias présentent de grandes potentialités pour la pisciculture, en partie liées à l'extrême facilité de leur élevage, une reproduction naturelle aisée en captivité, une croissance rapide, une grande résistance au manque d'oxygène, aux agents chimiques, aux maladies et aux manipulations fréquentes [34]. Les tilapias du genre Oreochromis sont caractérisés par un com- portement d'incubation buccale maternelle des jeunes [52]. La grande efficacité de la reproduction des espèces de ce groupe éco-éthologique ainsi que leur maturité précoce constituent des avantages certains en pisciculture $[3,41,45]$. Cependant, en milieu confiné comme un étang, ces caractéristiques conduisent à une surpopulation rapide qui entraîne une diminution de la croissance par un manque de nourriture et d'oxygène [32]. L'existence chez les tilapias d'un dimorphisme

* Corresponding author, Adresse actuelle : Association réunionnaise pour le développement de l'aquaculture, route forestière de l'Étang-du-Gol, B.P. 16, 97427 Étang-Salé, La Réunion, France - Fax : 262265001. 
sexuel de la croissance jouant en faveur des mâles [32, $45,50]$ orienta les recherches vers l'obtention de populations monosexes mâles, dans le but de supprimer toute reproduction incontrôlée et d'améliorer les performances des systèmes de production par une augmentation de la croissance, une réduction de l'agressivité et une diminution du comportement de territorialité. Une des techniques étudiées pour la production de populations monosexes mâles repose sur le modèle de la détermination génétique du sexe des espèces du genre Oreochromis $[12,21,27]$. Cette technique s'appuie sur la possibilité d'inverser le sexe d'alevins encore indifférenciés par apport de stéroïdes dans l'alimentation [56]. L'espèce Oreochromis aureus est caractérisée par une homogamétie mâle (ZZ/ZW) [18]. Ainsi, l'inversion hormonale du sexe d'une descendance, avec une hormone féminisante (la 17 $\alpha$-éthynyloestradiol), permet l'obtention de pseudofemelles, caractérisées par un phénotype femelle mais un génotype mâle ZZ. L'étape suivante consiste à repérer les pseudofemelles et les femelles au sein de la population sexuellement inversée. Ce repérage est basé sur l'analyse du sex-ratio dans les descendances des femelles et des pseudofemelles. En effet, le croisement d'une femelle normale (génotype WZ) avec un mâle normal (génotype $\mathrm{ZZ}$ ) donne en théorie $50 \%$ de mâles (génotype $Z Z$ ) et $50 \%$ de femelles (génotype WZ), alors qu'une pseudofemelle (génotype ZZ) croisée avec un mâle normal (génotype ZZ) donne, toujours en théorie, $100 \%$ de mâles (génotype $\mathrm{ZZ}$ ). Cette étape permet donc d'identifier des pseudofemelles de première génération (pseudofemelles F1) [33]. Afin d'augmenter rapidement les stocks de pseudofemelles, les alevins des descendances $100 \%$ mâle de pseudofemelles F1 sont sexuellement inversés avec la 17 $\alpha$-éthynylœstradiol. Des populations de pseudofemelles F2 sont ainsi obtenues. Sur le même principe, des générations successives de pseudofemelles (F3, F4...) peuvent être ainsi créées [33].

Chez les tilapias, de nombreuses études ont montré la fertilité d'individus issus d'une inversion hormonale du sexe $[21,27,28,29,33]$. Mais aucune étude ne s'est attachée à caractériser la reproduction d'individus présentant un phénotype opposé au génotype. Lors de travaux préliminaires [12], nous avions montré que le taux de reproduction des pseudofemelles F2 était plus faible que celui des femelles, lorsque les pseudofemelles et les femelles étaient mises en reproduction dans un même bassin. Ce résultat suggérait un effet du traitement hormonal ou du génotype sexuel sur la capacité de reproduction des pseudofemelles. Dans le cadre de la présente étude, nous avons déterminé plusieurs paramètres importants de la reproduction (taux de reproduction, fécondité, poids des cufs pondus) afin de rechercher un éventuel effet du génotype sexuel sur cette fonction biologique; le génotype sexuel étant défini comme l'ensemble des gènes possédés par un individu et agissant sur les caractères sexués. Pour cela, nous avons constitué trois groupes expérimentaux, l'un constitué uniquement de femelles, le second uniquement de pseudofemelles et le troisième de femelles et de pseudofemelles. Cette étude nous a également permis d'analyser l'influence de la température et de la durée d'éclairement sur l'évolution saisonnière de la reproduction des femelles et des pseudofemelles.

\section{MATÉRIEL ET MÉTHODE}

\subsection{Protocole d'obtention des pseudofemelles}

La souche Oreochromis aureus (Steindachner, 1864) utilisée dans cette étude est originaire de la station de Dor (Israël) et est acclimatée depuis 1978 à la Station d'aquaculture de l'université de Liège à Tihange (Belgique). Nous avons utilisé pour cette étude des pseudofemelles F3 (troisième génération), obtenues à la suite d'un traitement, 17 $\alpha$-éthynylœstradiol, de deux descendances monosexes mâles de pseudofemelles F2 (deuxième génération). Le traitement hormonal est appliqué à des alevins âgés de 9 jours après fécondation et pendant une durée de 40 jours. Les alevins sont nourris avec un aliment pour salmonidés ( $54 \%$ de protéines) dans lequel est incorporée l'hormone à une concentration de $150 \mathrm{mg}$ d'hormone $\cdot \mathrm{kg}^{-1}$ d'aliment. L'hormone est préalablement dissoute dans de l'éthanol à $95 \%$ puis mélangée à l'aliment $\left(800 \mathrm{~mL} \cdot \mathrm{kg}^{-1}\right.$ d'aliment). L'aliment est ensuite maintenu à l'air ambiant jusqu'à évaporation complète de l'éthanol. Pendant la période d'inversion, la ration alimentaire est déterminée d'après la formule suivante : ration $=0,18 \times$ durée $^{1,16}$ (g pour 100 poissons Mélard, données non publiées). L'aliment est distribué au moyen d'un nourrisseur automatique sur une période de 12 heures. Les poissons sont élevés dans des bassins de $1,0 \mathrm{~m}^{2} / 0,3 \mathrm{~m}^{3}$ d'un circuit fermé thermorégulé à $27^{\circ} \mathrm{C}$. Après la période d'inversion, les poissons reçoivent un aliment non traité. Jusqu'à la maturité sexuelle, ils sont élevés dans des bassins de $4 \mathrm{~m}^{2} / 1,6 \mathrm{~m}^{3}$ alimentés par de l'eau à $23-27^{\circ} \mathrm{C}$.

\subsection{Constitution et suivi des groupes expérimentaux}

Trois groupes de géniteurs, comprenant chacun trois mâles, sont constitués en janvier 1994 : les groupes 1 et 2 sont composés respectivement de 20 femelles et 20 pseudofemelles, le groupe 3 contient 10 femelles et 10 pseudofemelles. Pour la constitution des groupes, la sélection des femelles et des pseudofemelles est basée sur l'émission d'ovocytes suite à une pression abdominale. Au début de l'expérience, les géniteurs (femelles, pseudofemelles et mâles) se reproduisaient pour la première fois et étaient alors âgés de sept mois. Tous les individus sont identifiés par une marque magnétique («Pit Tag ») implantée dans la musculature dorsale. Les géniteurs sont pesés individuellement en début ( 5 janvier) et en fin ( 22 décembre) d'expérience. Les poids (corporels) moyens initiaux et finaux des femelles et des pseudofemelles des trois groupes sont présentés dans le tableau $I$. Les trois groupes de géniteurs sont maintenus chacun dans un bassin de 
Tableau I. Poids vif moyen des femelles et des pseudofemelles Oreochromis aureus dans les trois groupes expérimentaux.

Table I. Mean body weight $(\mathrm{g} \pm \mathrm{SD}$ ) of females and pseudofemales Oreochromis aureus in the three experimental batches.

\begin{tabular}{lccccc}
\hline & Groupe 1 & Groupe 2 & & Groupe 3 \\
\cline { 2 - 3 } \cline { 5 - 6 } & Femelles & Pseudofemelles & & Femelles & Pseudofemelles \\
\hline Nombre de géniteurs & 20 & 20 & 10 & 10 \\
Poids initial $(\mathrm{g})$ & $232 \pm 48$ & $256 \pm 64$ & $235 \pm 46$ & $248 \pm 77$ \\
Poids final $(\mathrm{g})$ & $427 \pm 70$ & $473 \pm 84$ & & $417 \pm 41$ & $462 \pm 41$ \\
\hline
\end{tabular}

$4 \mathrm{~m}^{2} / 1,6 \mathrm{~m}^{3}$, soit 6 individus $\cdot \mathrm{m}^{-2}$. Les bassins sont alimentés par l'eau des circuits de refroidissement de la centrale nucléaire de Tihange [34]. La température moyenne dans les bassins de reproduction varie de $24,3{ }^{\circ} \mathrm{C}$ en février à $27,2^{\circ} \mathrm{C}$ en juillet (figure 1 ). Le taux d'oxygène est maintenu entre 5,0 et $8,0 \mathrm{mg} \cdot \mathrm{L}^{-1}$. Les poissons sont soumis au régime photopériodique naturel (figure 1). Les poissons sont nourris à satiété avec des granulés flottants (34\% de protéines) deux fois par jour.

Une fois par semaine, les aufs et les alevins sont prélevés de la cavité buccale des femelles et des pseudofemelles des trois groupes. Les individus qui ont pondu sont ensuite pesés individuellement (au gramme près) et identifiés grâce à leur marque. Les cufs sont comptés et pesés (à $0,01 \mathrm{~g}$ près), les alevins sont comptés. La fécondité absolue est définie dans cette étude comme étant le nombre d'œufs ou d'alevins pondus et incubés par un géniteur. La fécondité relative correspond à la fécondité absolue ramenée à un kilogramme de poisson et est calculée sur la base du poids du géniteur lors du contrôle. Le taux de reproduction représente le nombre de pontes par individu au cours de la période expérimentale.

\section{RÉSULTATS}

\subsection{Influence de la température et de la durée d'éclairement sur la reproduction}

Les premières pontes de femelles et de pseudofemelles sont observées en mars. Les femelles et les pseudofemelles présentent une évolution saisonnière des pontes similaire $\left(\chi^{2}=10,0, P>0,05, \mathrm{ddl}=5\right)$, avec un pic en juin lorsque la température moyenne $\left(27,2^{\circ} \mathrm{C}\right)$ et la durée d'éclairement (16 heures) sont maximales (figure l). Par la suite, le nombre mensuel de ponte décroît jusqu'au mois de novembre et un arrêt des reproductions est observé en décembre. La diminution du nombre de pontes (de juillet à novembre) dans les différents groupes intervient lorsque la durée d'éclairement diminue. Au cours de cette même période, la température moyenne dans les bassins est comprise entre 27,0 et $27,2^{\circ} \mathrm{C}$. En revanche, en durée d'éclairement croissante, des pontes sont observées en avril dans les trois groupes alors que la température moyenne n'est que de $24,6^{\circ} \mathrm{C}$.

\subsection{Taux de reproduction}

Le nombre total de pontes récoltées dans les groupes 1 et 2 est respectivement de 138 et 94 . Dans le groupe 3 , le nombre de pontes des femelles $(n=64)$ est supérieur à celui des pseudofemelles $(n=6)$ (tableau II). Le nombre de pontes par individu varie de 0 à 16 chez les femelles et de 0 à 9 chez les pseudofemelles.

$\mathrm{Au}$ cours de la saison de reproduction, le taux de reproduction est de 6,9 pontes par femelle (groupe 1) et de 4,7 pontes par pseudofemelle (groupe 2) (différence non significative, test $t, t=2,18 ; P>0,05$, $\mathrm{ddl}=38$ ) (tableau II). Dans le groupe 3, la différence est significative avec une moyenne de 6,4 pontes par femelle et 0,6 par pseudofemelle (Mann et Whitney, $\mathrm{U}=4, P<0,05, \mathrm{n}=10 ; \mathrm{m}=10$ ). Entre les pseudofemelles du groupe 2 et du groupe 3 , la différence est

Tableau II. Taux de reproduction, fécondité, poids moyen des cufs des femelles et des pseudofemelles Orechromis aureus dans les trois groupes expérimentaux.

Table II. Spawning rate, fecundity, mean egg weight of females and pseudofemales Orechromis aureus in the three experimental batches.

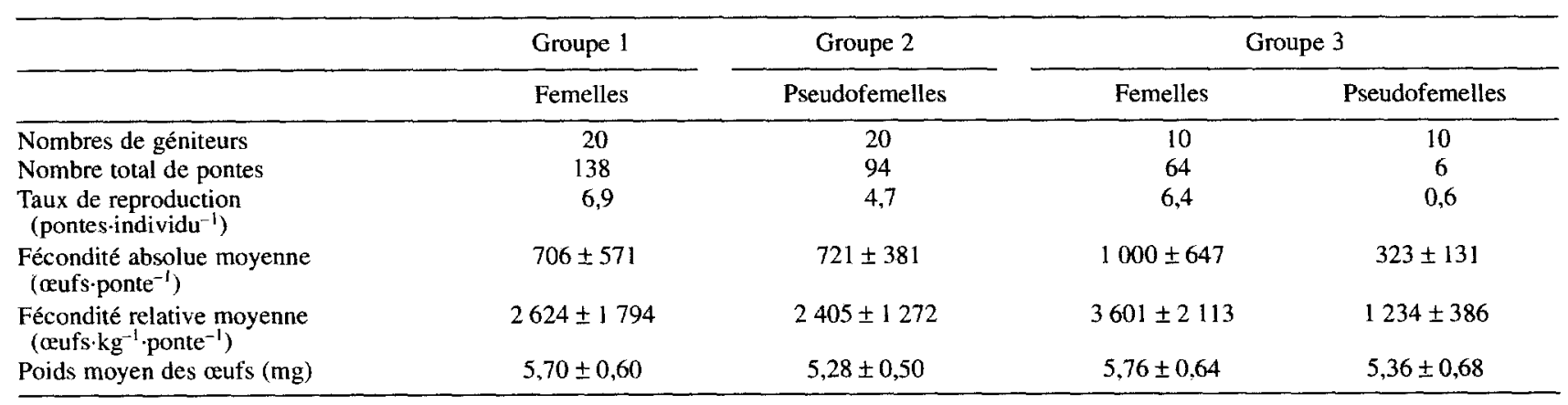

Aquat. Living Resour. 11 (3) (1998) 
Nombre de pontes
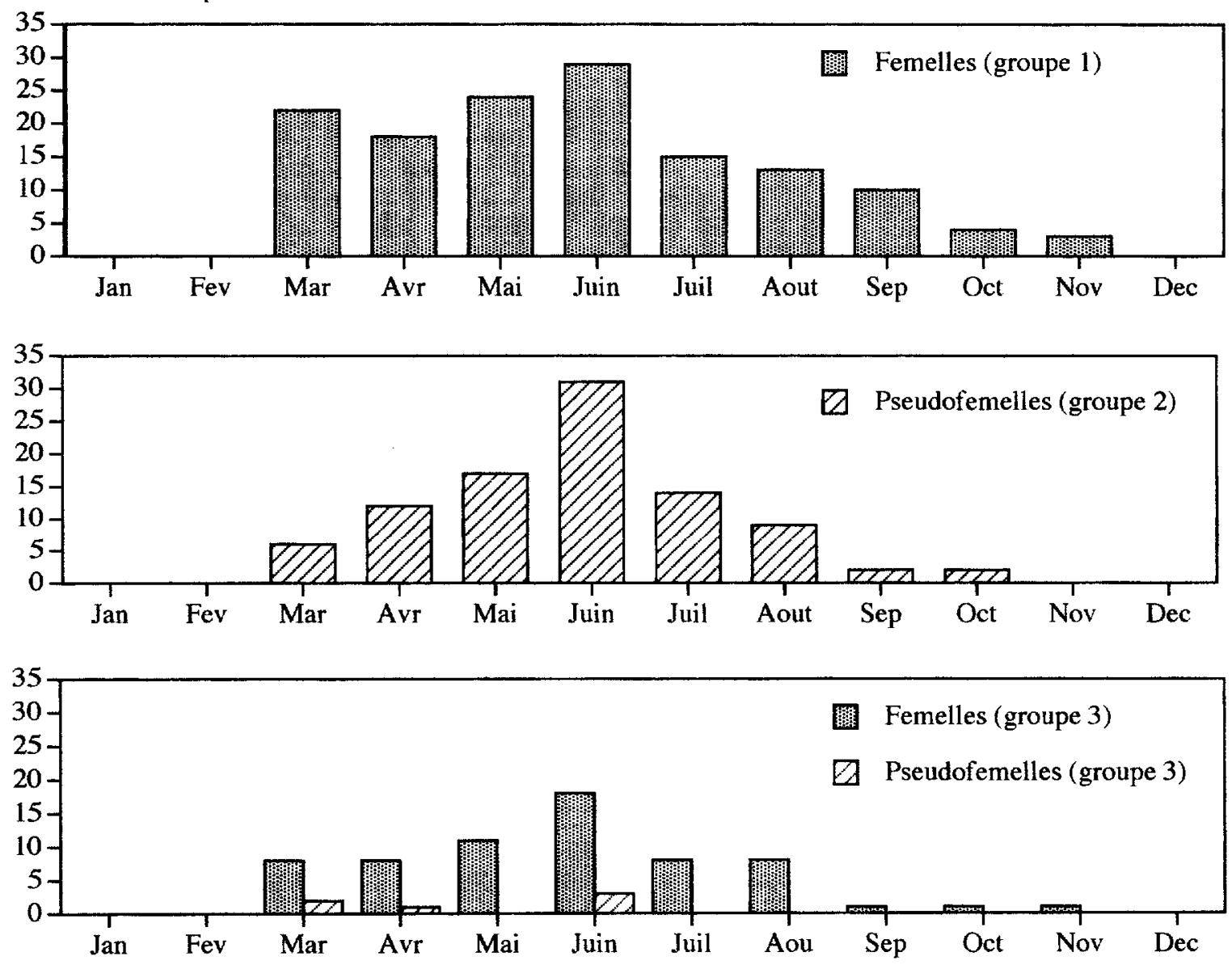

Durée d'éclairement (heures)

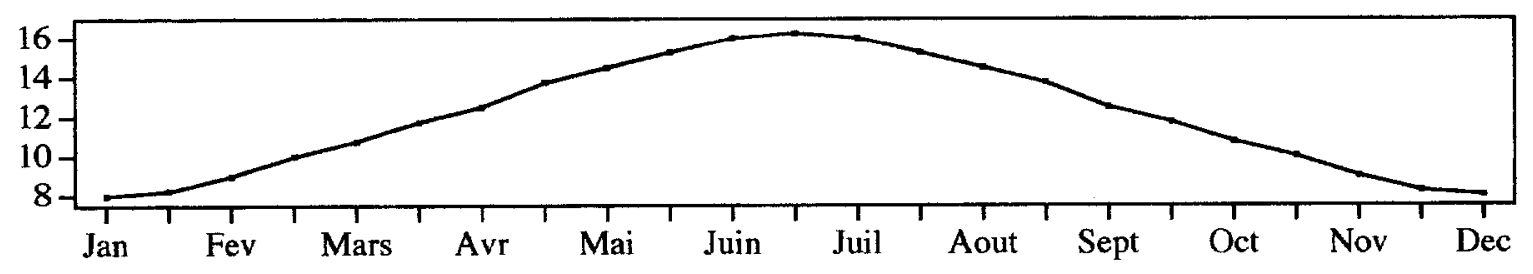

\section{Température $\left({ }^{\circ} \mathrm{C}\right)$}

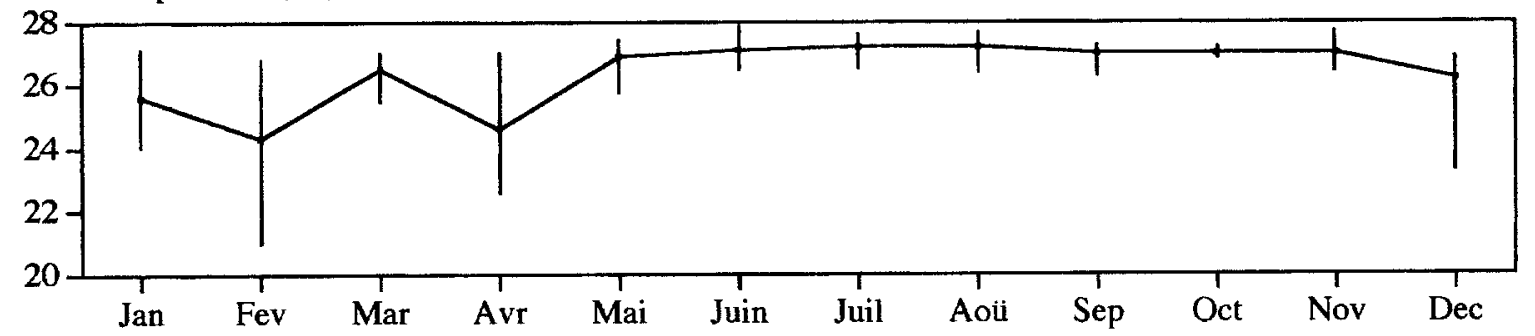

Figure 1. Évolution saisonnière de la répartition mensuelle des pontes de femelles et de pseudofemelles Oreochromis aureus dans les trois groupes expérimentaux, comparée à l'évolution de la température $\left({ }^{\circ} \mathrm{C}\right)$ et de la durée d'éclairement (h).

Figure 1. Seasonal evolution of the monthly distribution of spawnings of females and pseudofemales Oreochromis aureus in the three experimental batches, compared to evolution of temperature $\left({ }^{\circ} \mathrm{C}\right)$ and day length $(\mathrm{h})$ 
également significative, avec respectivement 4,7 et 0,6 pontes (Mann et Whitney, $\mathrm{U}=22, P<0,05, \mathrm{n}=10$; $\mathrm{m}=20$ ).

$\mathrm{Au}$ cours de la saison de reproduction, toutes les femelles et seulement $40 \%$ des pseudofemelles (soit 4 individus) du groupe 3 ont pondu au moins une fois (différence significative $\chi^{2}=85,7, P<0,05$, ddl = 1). En revanche dans le groupe 2 , où les pseudofemelles ne sont pas en présence de femelles, $90 \%$ des individus ont pondu (différence significative entre les pseudofemelles des groupes 2 et $3, \chi^{2}=55,0, P<0,05$, $\mathrm{ddl}=1$ ). Dans le groupe $1,95 \%$ des femelles ont pondu au moins une fois.

\subsection{Fécondité}

La fécondité absolue par ponte varie de 82 à 3132 œufs chez les femelles et de 26 à $2864 \mathrm{chez}$ les pseudofemelles. La fécondité absolue moyenne des femelles du groupe 1 et des pseudofemelles du groupe 2 est respectivement de $706 \pm 571$ et de $721 \pm$ 381 oufs ponte ${ }^{-1}$ (test $\mathrm{t}, t=0,23, P>0,05, \mathrm{ddl}=228$ ) (tableau II). En revanche, les femelles du groupe 3 présentent une fécondité absolue $(1000 \pm 647$ œufs. ponte $^{-1}$ ) significativement supérieure à celle des femelles du groupe 1 (test $\mathrm{t}, t=3,25, P<0,05$, ddl $=$ 198) et des pseudofemelles du groupe 2 (test $t, t=3,38$, $P<0,05$, ddl $=154$ ). La fécondité absolue des pseudofemelles du groupe 3 ( $323 \pm 131$ cufs ponte $\left.^{-1}\right)$ est plus faible que celle des femelles du groupe 1 (706 \pm 571 cufs.ponte $\left.{ }^{-1}\right)$, des pseudofemelles du groupe $2(721 \pm$ 381 aufs.ponte ${ }^{-1}$ ) et des femelles du groupe 3 (1 $000 \pm$ 647 œufs.ponte $^{-1}$ ) (tableau II). Le faible nombre de pontes $(n=6)$ pour les pseudofemelles du groupe 3 implique de considérer avec précaution les résultats obtenus sur ces poissons.

La fécondité relative des femelles du groupe 1 et des pseudofemelles du groupe 2 sont respectivement de $2624 \pm 1794$ et $2405 \pm 1272$ œufs.ponte ${ }^{-1} \cdot \mathrm{kg}^{-1}$ (pas de différence statistique, test $\mathrm{t}, t=1,02, P>0,05$, $\mathrm{ddl}=230$ ). La fécondité relative des femelles du groupe 3 (3 $601 \pm 2113$ œufs $\cdot$ ponte $\left.{ }^{-1} \cdot \mathrm{kg}^{-1}\right)$ est significativement supérieure à celle des femelles du groupe 1 (test $\mathrm{t}, t=3,38, P<0,05$, ddl $=200$ ) et des pseudofemelles du groupe 2 (test $\mathrm{t}, t=4,41, P<0,05$, ddl $=$ 154) (tableau II). La fécondité relative des pseudofemelles du groupe $2\left(2405 \pm 1272\right.$ œufs.ponte ${ }^{-1}$. $\mathrm{kg}^{-1}$ ) est plus élevée que celle des pseudofemelles du groupe 3 ( $1234 \pm 386$ œufs.ponte $\left.{ }^{-1} \cdot \mathrm{kg}^{-1}\right)$.

Le poids moyen des aufs des femelles (groupes 1 et 3) n'est pas différent de celui des pseudofemelles (groupe 2) (groupe 1 contre/vs groupe 2 : test $t$, $t=1,22, P>0,05, \mathrm{ddl}=133$; groupe 3 contre $/ \mathrm{vs}$ groupe 2 : test $\mathrm{t}, t=0,83, P>0,05, \mathrm{ddl}=90$ ). Aucune relation entre le poids des ceufs et le poids des femelles (groupe $1: r=-0,016, P<0,01$; groupe $3: r=-0,247$, $P<0,01)$ ou le poids des pseudofemelles $(\mathrm{r}=-0,319$, $P<0,01)$ n'est mise en évidence. Le poids des œufs n'est pas corrélé à la fécondité absolue dans les trois groupes $(r=-0,152 ; r=-0,449, r=-0,297$, respectivement avec $P<0,01$ ) (tableau $I I$ ).

\section{DISCUSSION}

\subsection{Influence de la température et de la durée d'éclairement sur la reproduction}

D'après nos observations, la durée d'éclairement jouerait un rôle sur la reproduction des femelles et des pseudofemelles $O$. aureus. En effet, à partir du mois de juillet, parallèlement à la diminution de la durée d'éclairement, le nombre de pontes décroît, alors que les températures restent favorables à la reproduction (figure 1). Chez les tilapias, des pontes peuvent être observées dès que la température atteint le seuil de reproduction, évalué à $18^{\circ} \mathrm{C}$ pour $O$. mossambicus [52] et entre 20 et $22^{\circ} \mathrm{C}$ pour $O$. niloticus et $O$. aureus [32, 52]. Dans une précédente étude, nous avions également montré que la durée d'éclairement influence l'activité de reproduction chez $O$. aureus et $O$. niloticus : celle-ci est respectivement stimulée et inhibée par les longues et courtes périodes d'éclairement. Cette étude avait également confirmé l'inhibition totale de la reproduction chez $O$. aureus sous une courte durée d'éclairement, même avec une température favorable [4]. Dans les zones où les saisons sont fortement marquées (ce qui est le cas ici), le maximum de ponte est observé lors de la période la plus chaude et la plus ensoleillée [26, 46]. En Afrique du Sud, une corrélation entre l'augmentation de la température et l'accroissement de l'Indice Gonado Somatique (IGS) est observée chez $O$. mossambicus [8]. Il ressort de leurs observations que la température est le régulateur environnemental le plus significatif sur le développement des gonades. Ces auteurs mettent également en évidence un effet du $\mathrm{pH}$ sur la maturation des organes sexuels. L'influence de la température et de la durée d'éclairement sur l'activité de reproduction des tilapias a été montrée chez Tilapia leucostica $[19,46]$, T. macrocephala [1], T. zillii [47]. Chez O. macrochir, la maturation des femelles est déclenchée par les variations de température [30]. Les facteurs environnementaux (température, durée d'éclairement, $\mathrm{pH}$, stabilité du milieu de vie, disponibilité de l'aliment...) ont certes un rôle important dans la maturation des organes sexuels et dans le déclenchement des pontes chez les tilapias. Cependant, des facteurs physiologiques individuels, les interactions sociales $[17,20]$ mais aussi l'alimentation $[6,7]$ peuvent également réguler les pontes et engendrer des variations saisonnières indépendamment des facteurs environnementaux. Ces observations conduisent [1] à définir, chez T. macrocephala, trois types de facteurs influençant la reproduction: (a) un facteur interne ou physiologique, (b) une stimulation par le contact visuel avec le partenaire, (c) des facteurs environnementaux et principalement la température et la durée d'éclairement. Dans notre étude, l'évolution du nombre de pontes des femelles et des pseudofemelles 
au cours de la saison peut effectivement être mise en relation avec la durée d'éclairement mais un phénomène d'épuisement des géniteurs ne peut être exclu pour expliquer la diminution des pontes à partir des mois de septembre-octobre. À ce propos, des auteurs $[1,37]$ ont observé une baisse des performances, suite à une période de reproduction de 4 à 6 mois, même avec des facteurs environnementaux favorables. Une possible fatigue métabolique suite à la période de reproduction est évoquée [36]. Comme nous le verrons ulté-rieurement, le prélèvement systématique des progé-nitures dans la bouche des géniteurs augmente la fréquence de ponte $[48,49,53]$ ce qui, de ce fait, peut accélérer le phénomène de fatigue métabolique évoqué par Miller [36] et expliquer la diminution des pontes en fin de saison dans les trois groupes. Il ressort toutefois de nos résultats que les femelles et les pseudofemelles présentent un profil saisonnier de ponte similaire.

\subsection{Taux de reproduction}

Dans le groupe composé de femelles et de pseudofemelles, le taux de reproduction des pseudofemelles est plus faible que celui des femelles alors qu'aucune différence significative n'est observée lorsque les femelles et les pseudofemelles sont mises en reproduction dans des structures d'élevage distinctes. La présence de femelles aurait donc un effet négatif sur le taux de reproduction des pseudofemelles. Meriwether et Shelton [35] attribuaient le faible taux de reproduction chez les pseudofemelles $O$. aureus à une inversion hormonale incomplète de la gonade et donc à une nonfonctionnalité de cette dernière chez certains individus. Cette hypothèse ne peut toutefois expliquer nos observations. En effet, l'absence de différence entre la fécondité des femelles du groupe 1 et des pseudofemelles du groupe 2 montre que l'inversion hormonale du sexe n'affecte pas la gamétogenèse des pseudofemelles. L'explication de ce phénomène pourrait résider dans le comportement de reproduction des pseudofemelles. Une analyse du comportement a été réalisée avec des pseudofemelles de la même origine que celles utilisées dans cette étude. En présence de femelles, les pseudofemelles sont plus agressives et dominantes visà-vis des femelles [42]. De plus, la présence dans un aquarium, d'un mâle isolé derrière une cloison, induit une diminution de l'agressivité des femelles mais n'affecte pas l'agressivité des pseudofemelles, en comparaison, avec les résultats obtenus sans le mâle [39]. Dans le groupe 3, les mâles pourraient donc se reproduire préférenciellement avec les individus les moins agressifs, donc les femelles. Cette hypothèse expliquerait la différence de taux de reproduction constatée entre les femelles et les pseudofemelles dans le groupe 3. En période de reproduction, l'agressivité et la territorialité sont caractéristiques du sexe mâle chez les espèces du genre Oreochromis. La plus forte agressivité des pseudofemelles pourrait donc être mise en relation avec leur génotype mâle $(\mathrm{ZZ})$.
Nous avons aussi observé une grande variabilité individuelle du nombre de ponte par individu. En effet, certaines femelles n'ont jamais pondu au cours de l'étude alors que d'autres ont pondu jusqu'à 16 fois. Chez les pseudofemelles, la variation s'échelonne de 0 à 9 pontes. Ces observations sont en accord avec les données recueillies sur les tilapias : 2 et 7 pontes par an chez $T$. leucostica [55] et $T$. esculenta [9]; 1 à 14 pontes par an chez $T$. macrocephala [1], 4 à 5 pontes par an chez $O$. mossambicus [43] et $T$. variabilis [15], 3 à 8 pontes [22] avec une moyenne de 3 à 4 pontes par an [57] chez $O$. aureus. Une population pourrait comporter des femelles dominantes qui se reproduiraient plus fréquemment que les autres [31, 37]. La moyenne de ponte par femelle dans notre étude est de 6,4 (groupe 3) et 6,9 (groupe 1) donc supérieure à celle observée pour la même espèce par Yashouv [57]. Dans nos conditions expérimentales, les æufs ou les alevins sont systématiquement prélevés de la bouche des géniteurs lors du contrôle hebdomadaire, or le prélèvement des œufs augmente la fréquence de ponte chez O. niloticus [49, 53] et chez O. mossambicus [48]. Chez $O$. niloticus, l'intervalle de temps entre deux pontes successives est de 27 jours pour des femelles qui incubent normalement et de 15 jours pour des femelles dont le retrait des œufs est effectué un jour après la ponte [49]. Un effet inhibiteur des soins parentaux sur le développement de l'ovaire est également mis en évidence [49]. Chez $O$. mossambicus, une période d'incubation de 25 jours a été déterminée [48]. La nouvelle ovulation peut ensuite avoir lieu 15 jours après la libération des alevins par la femelle. En revanche, si les œufs sont recrachés, en général un jour après la fécondation, la nouvelle ovulation aura lieu 25 jours après la première ponte [48]. Le nombre élevé de pontes par femelle (groupes 1 et 3 ) et par pseudofemelle (groupe 2) $O$. aureus résulte probablement du prélèvement systématique des œufs après la ponte qui induit une augmentation de la fréquence de pontes.

\subsection{Fécondité absolue}

Dans notre étude, les données obtenues sur $O$. aureus montrent que le traitement hormonal n'affecte pas la fécondité des pseudofemelles. En effet, ces dernières (groupe 2) présentent des fécondités absolue et relative équivalentes à celles des femelles (groupe 1). La forte variabilité de la fécondité absolue est observée chez plusieurs espèces de tilapia : 320 à 1328 œufs par ponte chez $O$. leucosticus [46], 325 à 4392 chez O. aureus [10], 342 à 3504 chez O. niloticus [32], 69 à 1850 chez Sarotherodon galilaeus [5], 674 à 7009 chez T. zillii [11], 368 à 718 chez $S$. melanotheron [24]. Avec une moyenne de 719 aufs chez $O$. aureus, Dadzie [10] obtient des données comparables à nos résultats. Les différences de fécondité constatées entre nos différents groupes sont plus difficiles à interpréter. En effet, les femelles du groupe 3 présentent une fécondité absolue supérieure à celle des pseudofemelles du groupe 2, mais également par rap- 
port aux femelles du groupe 1. En effet, tous les groupes étaient élevés dans les mêmes conditions (température, durée d'éclairement, alimentation, etc.). Ces différences entre les groupes de géniteurs pourraient résulter d'une variabilité individuelle qui pourrait être mise en relation avec les réserves énergétiques et le niveau d'alimentation $[38,51]$.

La fécondité chez les tilapias est corrélée à la taille et/ou au poids des géniteurs et ce tant en milieu naturel $[2,14,25,52]$ que dans des conditions expérimentales $[44,54]$. Une forte corrélation entre la taille, le poids et la fécondité est mise en évidence chez $O$. niloticus [16, 32] Cependant, pour des individus de même âge ou se situant dans une même gamme de poids (ce qui est le cas ici), aucune relation marquée n'existe $[6,7$, notre étude].

\subsection{Poids des ceufs}

Chez les tilapias, l'existence d'une relation entre le poids des œufs et les autres caractères est controversée. Aucune relation n'est observée avec la taille chez $S$. melanotheron [13] ou le poids des géniteurs chez $T$. zillii [11]. En revanche, une augmentation de la taille des œufs avec l'augmentation de la taille du poisson est observée chez $S$. melanotheron [40]. Cette observation est confirmée par Legendre et Ecoutin [23] qui constatent que le poids ovocytaire tend à augmenter avec le poids des femelles juqu'à un poids corporel de $100 \mathrm{~g}$, pour ensuite se stabiliser. Chez $O$. aureus, une diminution du diamètre des ovocytes au cours de la saison de reproduction est mise en évidence [44]. Dans une étude préliminaire, nous avions également mis en évidence une relation entre le rang de ponte et le poids moyen des cufs chez les femelles et des pseudofemelles F2 (Desprez, données non publiées). Dans notre étude, la variation du poids des oufs au cours des pon- tes successives est faible et aucune relation n'apparaît avec le poids des géniteurs, la fécondité absolue ou le rang de ponte. L'impossibilité de généraliser un modèle d'évolution du poids moyen des œufs en fonction des différents paramètres biologiques pourrait s'expliquer par une variabilité individuelle.

\section{CONCLUSION}

Nos résultats montrent que les pseudofemelles présentent une fécondité, un poids moyen des œufs pondus et un profil saisonnier de ponte identique à celui des femelles. Les pseudofemelles paraissent sensibles aux mêmes stimuli environnementaux pour le déclenchement de la maturation sexuelle et de la reproduction. Toutefois, la présence de femelles et de pseudofemelles dans la même enceinte d'élevage induit un effet négatif sur le taux de reproduction des pseudofemelles. Ce résultat est mis en relation avec un taux d'agressivité plus élevé chez les pseudofemelles (génotype mâle $\mathrm{ZZ}$ ) que chez les femelles (génotype femelle $\mathrm{ZW}$ ). Notre étude tend donc à montrer qu'une inversion du sexe en utilisant l'hormone $17 \alpha$-éthynylœstradiol, appliquée pendant la période de la différenciation sexuelle chez $O$. aureus, permet la formation d'ovaires fonctionnels et n'altère pas la gamétogenèse. L'inversion hormonale n'induit toutefois pas une féminisation complète de l'individu, notamment au niveau du comportement sexuel. En effet, des caractéristiques comportementales typiquement mâles (notamment l'agressivité) s'expriment toujours chez les pseudofemelles. Cette observation suggère que le comportement de reproduction chez Oreochromis aureus résulterait de l'interaction de facteurs, hormonaux notamment, liés à l'expression du phénotype sexuel et de facteurs génétiques liés à l'expression du génotype sexuel.

\section{Remerciements}

Ces recherches ont été réalisées grâce au soutien financier d'Electrabel et de la société Piscimeuse S.A.

\section{RÉFÉRENCES}

[1] Aronson L.R., Factors influencing the spawning frequency in the female cichlid fish Tilapia macrocephala, Am. Mus. Novit. 1484 (1951) 1-26.

[2] Babiker M.M., Ibrahim H., Studies on the biology of reproduction in the cichlid Tilapia nilotica (L.): gonadal maturation and fecundity, J. Fish Biol. 14 (1979) $437-$ 448.

[3] Baroiller J.F., Jalabert B., Contribution of research in reproductive physiology to the culture of tilapias, Aquat. Living Resour. 2 (1989) 105-116.
[4] Baroiller J.F., Desprez D., Carteret Y., Tacon P., Borel F., Hoareau M.C., Mélard C., Jalabert B., Influence of environmental and social factors on the reproductive efficiency in three tilapia species, Oreochromis niloticus, $O$. aureus and the red tilapia (Red Florida strain), in: Fitzimmons K. (Ed.), Proc. 4th Int. Symp. Tilapia in Aquaculture, Orlando, 1997, pp. 238-252.

[5] Blay Jr. J., Fecundity and spawning frequency of Sarotherodon galilaeus in a concrete pond, Aquaculture 25 ( 1981 ) 95-99.

[6] Bromage R.N., Roberts R.J., Broodstock management and egg and larval quality, in: Bromage R.N., Roberts R.J. (Eds.), Blackwell Science Ltd Oxford, 1995, 424 p. 
[7] Cissé A., Effect of varying protein level on spawning frequency and growth of Sarotherodon melanotheron, in: Pullin R.S.V., Bhukaswan T., Tonguthai K., Maclean J.L. (Eds.), Proc. 2nd Inter. Symp. Tilapia in Aquaculture, Bangkok, Thailand, ICLARM Conf. Proc. 15, 1988, pp. 329-333.

[8] Cornish D.A., Smit G.L., The correlation between environmental factors and the reproduction of Oreochromis mossambicus, Water S. Afr. 21 (1995) 259-263.

[9] Cridland C.C., Breeding studies on Tilapia zillii and Tilapia nigra, East Afr. Fish. Res. Organisation Ann. Rep., 1961, pp. 29-32.

[10] Dadzie S., Laboratory experiment on the fecundity and frequency of spawning in Tilapia aurea, Bamidgeh 22 (1970) 14-18.

[11] Dadzie S., Wangila B.C.C., Reproductive biology, length-weight relationship and relative condition of pond raised Tilapia zillii, J. Fish Biol. 17 (1980) 243253.

[12] Desprez D., Mélard Ch., Philippart J.C., Production of a high percentage of male offspring with $17 \alpha$-ethynylestradiol sex-reversed Oreochromis aureus. II. Comparative reproductive biology of female and F2 pseudofemales and large scale production of male progeny, Aquaculture 130 (1995) 35-41.

[13] Eyeson K.N., Stunting and reproduction in pond-reared Sarotherodon melanotheron, Aquaculture 31 (1983) 257-267.

[14] Fagade S.O., Observations on the biology of two species of Tilapia from the Lagos lagoon, Nigeria, Bull. Inst. Fr. Afr. Noire 41 (1979) 627-651.

[15] Fryer G., Observations on the biology of the cichlid fishes Tilapia variabilis (Boulenger) in the northern waters of Lake Victoria (East Africa), Rev. Zool. Bot. Afr. 64 (1961) 1-33.

[16] Fryer G., Isles T.D., The cichlid fishes of the great lakes of Africa: their biology and evolution, Oliver and Boyd (Eds.), Edinburgh, 1972, 641 p.

[17] Gautier J.Y., Lefaucheux B., Foraste M., Interactions entre comportement incubateur et cycles sexuels, in: Pullin R.S.V., Lazard J., Legendre M., Amon Kotias J.B., Pauly D. (Eds.), Proc. 3rd Int. Symp. Tilapia in Aquaculture, Abidjan, Côte d'Ivoire, ICLARM Conf. Proc. 41, 1996, pp. 308-313.

[18] Guerrero R.D., Use of androgens for the production of all male Tilapia aurea (Steindachner), Trans. Am. Fish. Soc. 104 (1975) 342-348.

[19] Hyder M., Gonadal and reproductive patterns in Tilapia leucostica (Teleostei, Cichlidae) in an equatorial lake, Lake Naivasha (Kenya), J. Zool. London 162 (1970) $179-195$.

[20] Jalabert B., Zohar Y., Reproductive physiology in Cichlid fishes, with particular reference to Tilapia and Sarotherodon, in: Pullin R.S.V., Lowe McConnell R.H. (Eds.), Proc. Biol. Cult. Tilapias, Manila, Philippines, ICLARM Conf. Proc. 7, 1982, pp. 129-140.

[21] Jensen G.L., Shelton W.L., Effects of estrogens on Tilapia aurea: implications for production of monosex genetic male tilapia, Aquaculture 16 (1979) 233-242.

[22] Lee J.C., Reproduction and hybridization of three cichlid fishes, Tilapia aurea (Steindachner), $T$. hornorum (Trewavas) and T. nilotica (Linnaeus) in aquaria and plastic pools, Ph.D. thesis, Auburn University, Alabama, USA, 1979, 84 p.

[23] Legendre M., Ecoutin J.M., Aspects de la stratégie de reproduction de Sarotherodon melanotheron (Rüppel, 1852): comparaison entre une population naturelle (lagune Ebrié, Côte d'Ivoire) et différentes populations d'élevage, in: Pullin R.S.V., Lazard J., Legendre M., Amon Kotias J.B., Pauly D. (Eds.), Proc. 3rd Int. Symp. Tilapia in Aquaculture, Abidjan, Côte d'Ivoire, ICLARM Conf. Proc. 41, 1996, pp. 139-152.

[24] Legendre M., Trebaol L., Efficacité de l'incubation buccale et fréquence de ponte de Sarotherodon melanotheron (Rüppel, 1852) en milieu d'élevage (lagune Ebrié, Côte d'Tvoire), in: Pullin R.S.V., Lazard J., Legendre M., Amon Kotias J.B., Pauly D. (Eds.), Proc. 3rd Int. Symp. Tilapia in Aquaculture, Abidjan, Côte d'Ivoire, ICLARM Conf. Proc. 41, 1996, pp. 152-166.

[25] Lowe-Mc Connel R.H., The fecundity of tilapia species, East Afr. Agric. J. 21 (1955) 45-51.

[26] Lowe-McConnel R.H., Observation on the biology of Tilapia nilotica (L.) in East African waters, Rev. Zool. Bot. Afr. 57 (1958) 129-170.

[27] Mair G.C., Abucay J.S., Skibinski D.O.F., Abella T.A., Beardmore J.A., Genetic manipulation of sex ratio for the large scale production of all-male tilapia, Oreochromis niloticus, Can. J. Fish. Aquat. Sci. 54 (1997) 396404.

[28] Mair G.C., Scott A.G., Penman D.J., Skibinski D.O.F., Beardmore J.A., Sex determination in the genus Oreochromis. 1. Sex reversal, gynogenesis and triploidy in O. niloticus (L), Theor. Appl. Genet. 82 (1991) 144 152.

[29] Mair G.C., Scott A.G., Penman D.J., Skibinski D.O.F., Beardmore J.A., Sex determination in the genus Oreochromis. 2. Sex reversal, hybridisation, gynogenesis and triploidy in $O$. aureus (Steindachner), Theor. Appl. Genet. 82 (1991) 153-160.

[30] Marshall B.E., Observations on the breeding biology of Sarotherodon macrochir (Boulenger) in Lake Mc Ilwaine, Rhodesia, J. Fish Biol. 14 (1979) 419-424.

[31] McBay K.R., The biology of Tilapia nilotica, Proc. Ann. Conf. South-East Assoc. Game Fish Comm. 15 (1961) 208-218.

[32] Mélard C., Recherches sur la biologie d'Oreochromis niloticus L. (Pisces Cichlidae) en élevage expérimental: reproduction, croissance, bio-énergétique, Cah. Ethol. Appl. 6 (1986) 1-224.

[33] Mélard C., Production of a high percentage of male offspring with $17 \alpha$-ethynylestradiol sex reversed Oreochromis aureus. I. Estrogen sex reversal and production of F2 pseudofemales, Aquaculture 130 (1995) 25-34.

[34] Mélard C., Philippart J.C., Pisciculture intensive du tilapia Sarotherodon niloticus dans les effluents thermiques d'une centrale nucléaire en Belgique, in: Proc. World Symp. Aquaculture in heated effluents and recirculation systems, Stavanber, Norway, 1981, pp. 637-658.

[35] Meriwether F.H., Shelton W.L., Observation on aquarium spawning of estrogen treated and untreated Tilapia, Proc. Ann. Conf. S.E. Assoc. Fish Wild Life Agencies 34 (1986) 81-87.

[36] Miller A.H., The refractory period in light induced reproductive development of golden crowned sparrows, J. Exp. Biol. 109 (1984) 1-11. 
[37] Mires D., A study of the problems of the mass production of hybrid tilapia fry, In: Pullin R.S.V., Lowe McConnell R.H. (Eds.), Proc. Biol. Cult. Tilapias, Manila, Philippines, ICLARM Conf. Proc. 7, 1982, pp. 317-329.

[38] Mironova N.V., Energy expenditure on egg production in young Tilapia mossambica and the influence of maintenance conditions on their reproductive intensity, J. Ichthyol. 17 (1978) 627-633.

[39] Ovidio M., Etude éthologique et endocrinologique comparée de femelles et de pseudofemelles Oreochromis aureus (Steindachner, 1864) (Pisces, Cichlidae), mémoire de licence, Université de Liège, 1994, 49 p.

[40] Peters H.M., Eizahl, eigewicht und gelegeentwicklung in der gattung Tilapia (Cichlidae, teleostei), Int. Rev. Gesamt Hydrobiol. 48 (1963) 547-576.

[41] Philippart J.C., Ruwet J.C., Ecology and distribution of tilapias, in: Pullin R.S.V., Lowe McConnell R.H. (Eds.), Proc. Biol. Cult. Tilapias, Manila, Philippines, ICLARM Conf. Proc. 7, 1982, pp. 15-59.

[42] Poncin P., Ovidio M., Skoufas G., Gesquière V., Mélard C., Desprez D., Mol K., Byamungu N., Cuisset B., Kühn E.R., Philippart J.C., Ruwet J.C., Behavioural and endocrine study of Oreochromis aureus, with special reference to sex-reversed males, in: Goetz F.W., Thomas P. (Eds.), Proc. 5th Int. Symp. Reprod. Physiol. Fish, Austin, Texas, 1995, pp. 262-263.

[43] Riedel D., Some remarks on the fecundity of Tilapia (T. mossambica Peters) and its introduction into Middle Central America (Nicaragua) together with a first contribution towards the limnology of Nicaragua, Hydrobiologia 25 (1965) 357-388.

[44] Rojas Menocal R.L., Fecundidad de la tilapia Oreochromis aureus (Steindachner, 1864) en la presa Zaza, Rev. Lat. Acui. Lima Perù 3 (1988) 36-45.

[45] Ruwet J.C., Voss J., Hanon L., Micha J.C., Biologie et êlevage du tilapia, in: F.A.O. (Eds.), Proc. Inland Fish. Afr. No 4, Accra, Ghana, 1976, pp. 332-364.

[46] Siddiqui A.Q., Reproductive biology, length-weight relationship and relative condition of Tilapia leucostica
(Trewavas) in Lake Naivasha, Kenya, J. Fish Biol. 10 (1977) 251-260.

[47] Siddiqui A.Q., Reproductive biology of Tilapia zillii (Gervais) in Lake Naivasha, Kenya, Environ. Biol. Fishes 4 (1979) 257-262.

[48] Smith C.J., Haley S.R., Evidence of steroidogenesis in post ovulatory follicles of the tilapia Oreochromis mossambicus, Cell. Tissue Res. 247 (1987) 675-687.

[49] Tacon P., Ndiaye P., Cauty C., Le Menn F., Jalabert B., Reationship between expression of maternal behaviour and ovarian development in the mouthbrooding cichlid fish, Oreochromis niloticus, Aquaculture 146 (1996) 261-275.

[50] Toguyeni A., Fauconneau B., Boujard T., Fostier A., Kühn E.R., Mol K.A., Baroiller J.F., Feeding behaviour and food utilisation in tilapia, Oreochromis niloticus: effect of sex ratio and relationship with the endocrine status, Physiol. Behav. 62 (1997) 273-279.

[51] Townsend T.J., Wooton R.J., Effects of food supply on the reproductive of the convict cichlid Cichlasoma nigrofasciatum, J. Fish Biol. 24 (1984) 91-104.

[52] Trewavas E., Tilapia fishes of the genera Sarotherodon, Oreochromis et Danakilia, Cornell University press, New York, 1983, 583 p.

[53] Verdegem M.C., McGinty A.S., Effects of frequency of eggs and fry removal on spawning by Tilapia nilotica in hapas, Progress. Fish Cult. 49 (1987) 129-131.

[54] Watanabe W.O., Kuo C.M., Observation on the reproductive performance of Nile tilapia (Oreochromis niloticus) in laboratory aquaria at various salinity, Aquaculture 49 (1985) 315-323.

[55] Welcomme R.L., The relationship between fecundity and fertility in the mouthbrooding cichlid fish Tilapia leucostica, J. Zool. London 151 (1967) 453-468.

[56] Yamamoto T., Artificially induced sex-reversal in genotypic males of the Medaka (Oryzia latipes), J. Exp. Zool. 123 (1953) 571-594.

[57] Yashouv A., Biological data on Tilapia galilaea and Tilapia nilotica in fish ponds, Bamidgeh 10 (1958) 46-52. 